\title{
The Virtualisation of Network Functions and the Software Defined Network Improving the Economics of the Network
}

\author{
Sundar lyer \\ Strategist \& Account Chief Technologist, Communications, Media \& \\ Entertainment, Hewlett Packard Office of the CTO, Asia-Pacific \& \\ Japan
}

\begin{abstract}
Summary: A 'telco' or telecommunications service provider's network is both the source of its high cost base and source of differentiation. Telcos incur the high capital and operational costs of customised, vendor-proprietary hardware as they seek differentiation through network capacity addition, service quality and coverage improvement. These decisions to invest in the network are motivated by supply-side competition and demand - particularly cross-segment demand for data services, much of which is for "over-the-top" social networking and video streaming services that the telco can only partially monetise. This has created a dichotomy of margin erosion - a high cost to serve with diminishing returns on additional capacity investments.

However, an approach that blends IT, networking and data centre technologies promises to improve this equation, as evidenced by primary research, surveys and proof-of-concepts by Hewlett Packard. Network Functions Virtualisation (NFV) offers to immediately lower the capital and operational cost base by implementing a growing set of previously closed network platforms and functions on standard IT hardware. This will set the scene in the next few years for faster "software-defined network" (SDN) additions which will improve utilisation of network assets and allow lower-cost experiments to flex and scale capacity to improve network services and introduce new innovations. This article provides an introductory view of these emergent developments.
\end{abstract}

\section{Introduction}

In August 2011, in an essay written for the Wall Street Journal, Marc Andreessen, the founder of Netscape, Opsware and VC firm, Andreessen Horowitz, said, "Software is eating the world." (Andreessen, 2011). This article will make clear that this trend is manifest in the networks of telecommunication companies, or telcos. This emergent development, known as network functions virtualisation (NFV) and the software-defined network (SDN) is not a solution looking for a problem. Rather, the development was initiated by leading telcos 
around the world, (Chiosi et al, 2012) and it has gathered considerable momentum, moving from whitepapers to the lab to production networks. This article will focus on the economic drivers for NFV and SDN, drawing on the work of industry groups, standards bodies and primary research. It is important to note that NFV and SDN are complementary, but not mutually dependent. They can be combined for greater value to shift the classical network approach which is proprietary, cumbersome, and manual to a better economic model that is agile, standard and more automated.

\section{The Economics of Networks}

The telco's network is the source of its high cost base and source of differentiation. Telcos incur the high capital and operational costs of customised, vendor-proprietary hardware as they seek differentiation through network capacity addition, service quality and coverage improvement.

There are at least two reasons for this high cost base - demand that isn't fully monetisable, and supply-side inefficiencies associated with investments in customised, proprietary platforms.

The combination of these factors has created a dichotomy of margin erosion - a high cost to serve with diminishing returns on additional capacity investments.

\section{Network demand drivers}

Telcos, to remain competitive, must incur the high capital and operational costs of networks to cater to the rapid acceleration of demand, particularly for data services. Those that do not will face the churning of their customers to competitor networks.

As such, a "telco" or telecommunications service provider's network costs are often measured in the billions. For example, in Australia, Telstra (Ramli, 2014), Optus (Bingemann, 2014) and Vodafone (Bingemann, 2013) have all announced multi-year, billion dollar network investments.

Consider the impact on revenue and the requisite network capacity investments of the following statistics from the Australian Communications and Media Authority.

1. The increase in demand for data: 676,898 terabytes worth of data was downloaded during the June quarter of 2013, a "sobering" 59 per cent increase on the June quarter of 2012. (ACMA, 2013).

2. The centrality of mobility: 7.5 million Australians used the internet via their mobile phone during June 2013, an increase of 33 per cent (or ten percentage points) 
compared to June 2012 and a telling 510 per cent increase since June 2008. (스A, 2013).

3. The increasing amount of time and reliance on data services: At June 2013, 10.81 million Australians went online more than once a day-a seven per cent increase compared to the same time last year and a 72 per cent increase from June 2008.

4. Demand for OTT services: - 7.86 million Australians used "over the top" (OTT) content services such as catch-up TV, video on demand (e.g. Netflix) and IPTV in the six months to May 2013, an increase of 52 per cent compared to May last year. There were similarly spectacular increases in the use of OTT social messaging applications (e.g. Facebook-WhatsApp) and OTT VoIP services (e.g. Skype).

5. While there are no statistics publicly available for the erosion of Australian telco revenue by OTTs, global data trends are highly likely to be relevant in Australia. According to Ovum, OTT social messaging applications globally cost CSPs $\$ 13.9$ billion in lost SMS revenues in 2011, $\$ 32.5^{B}$ in 2013, and are predicted to cost $\$ 54$ billion by 2016. Additionally, by 2020, OTT VoIP providers will have cost the global telecoms industry $\$ 63$ billion in lost revenues. (Ovum, 2013)

In short, the situation is one of accelerating demand, but a slower rise in revenues due to OTTs capturing increasingly larger slices of revenue. This is compounded by historic pricing models which do not reflect changing cost structures.

\section{Supply-side drivers}

To date, network capacity investments to cater to the demand detailed above have entailed several capital inefficiencies attendant with customised, proprietary systems. These include:

1. Customised, closed interfaces between many network functions and their closely coupled management entities - this means that specific network elements are the preserve of a small number of network equipment suppliers, reducing competition and innovation. The price-performance trends of network equipment have not followed IT equipment.

2. Customised automation and operating procedures. This creates siloes of rare, and thus expensive, skills which in turn limits the agility of new service introduction, order-to-activate, and trouble-to-resolve processes and the modernisation of legacy network platforms. This places telcos at a competitive disadvantage vis-à-vis more nimble (over-the-top) OTT players. 
3. Increased power usage since network workloads are, in the most part, tied to dedicated hardware platforms. There is no flexibility in powering down and consolidating network functions on less hardware when that hardware is not in use.

4. Moreover, hardware-based appliances rapidly reach end of life, requiring much of the procure-design-integrate-deploy cycle to be repeated with little or no revenue benefit.

The logic would suggest, therefore, that the upward trajectory of data traffic growth (a proxy for network costs to meet capacity requirements) and the reduced gradient of revenue increases will create a revenue gap if no alternative paths are pursued. This is shown in Figure 1.

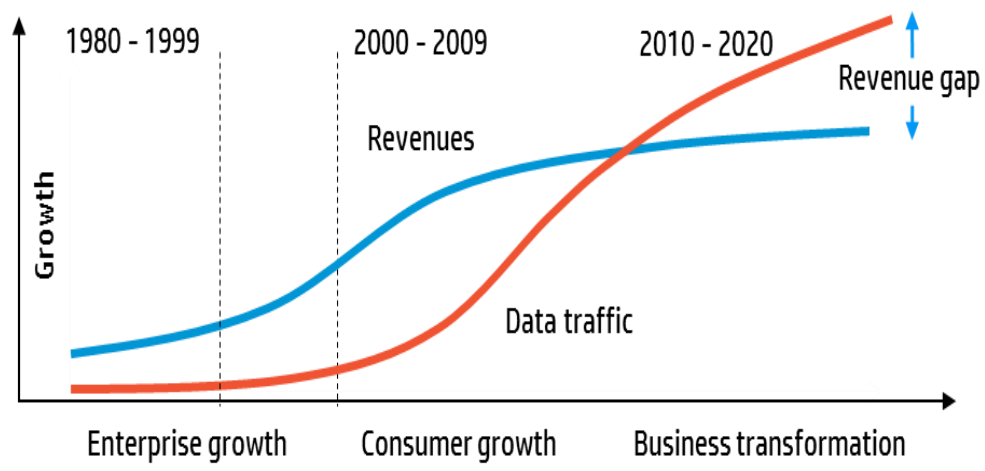

\section{Figure 1 - The Growing Revenue-Cost Gap for Telcos}

\section{For illustrative purposes only (not to scale)}

Fortunately, there are reasons to be more optimistic. At least one alternative model does exist, and it has gathered considerable momentum since 2012, when it was first mooted by 10+ Tier-1 global telcos. (Chiosi et al, 2012) That approach is known as network functions virtualisation (NFV) which, in turn, enables the software-defined network (SDN).

The impact of NFV and SDN will be to address the supply-side inefficiencies and allow greater service innovation, resulting in higher revenues.

\section{Network Functions Virtualisation}

NFV allows the movement away from the proprietary to the standard, from the cumbersome to the agile, from the manual to the automated, and from on-premise to the cloud.

According to the European Telecommunications Standards Institute, (ETSI, 2013) "Network Functions Virtualisation aims to transform the way that network operators architect networks by evolving standard IT virtualisation technology to consolidate many network equipment types on to industry standard high volume server, switches and storage, which could be located in a variety of NFVI-PoPs including data centres, network nodes and in 
end user premises." (ETSI, 2013) ETSI goes on to say that "in principle, all network functions and nodes may be considered for virtualisation and should be enabled by standards.”(ETSI, 2013).

Figure 2 describes the differences between the classical or traditional approach and the new model. This was first articulated by a consortium of network operators, themselves, precluding issues of solutions in search of problems. (Chiosi et al, 2012)

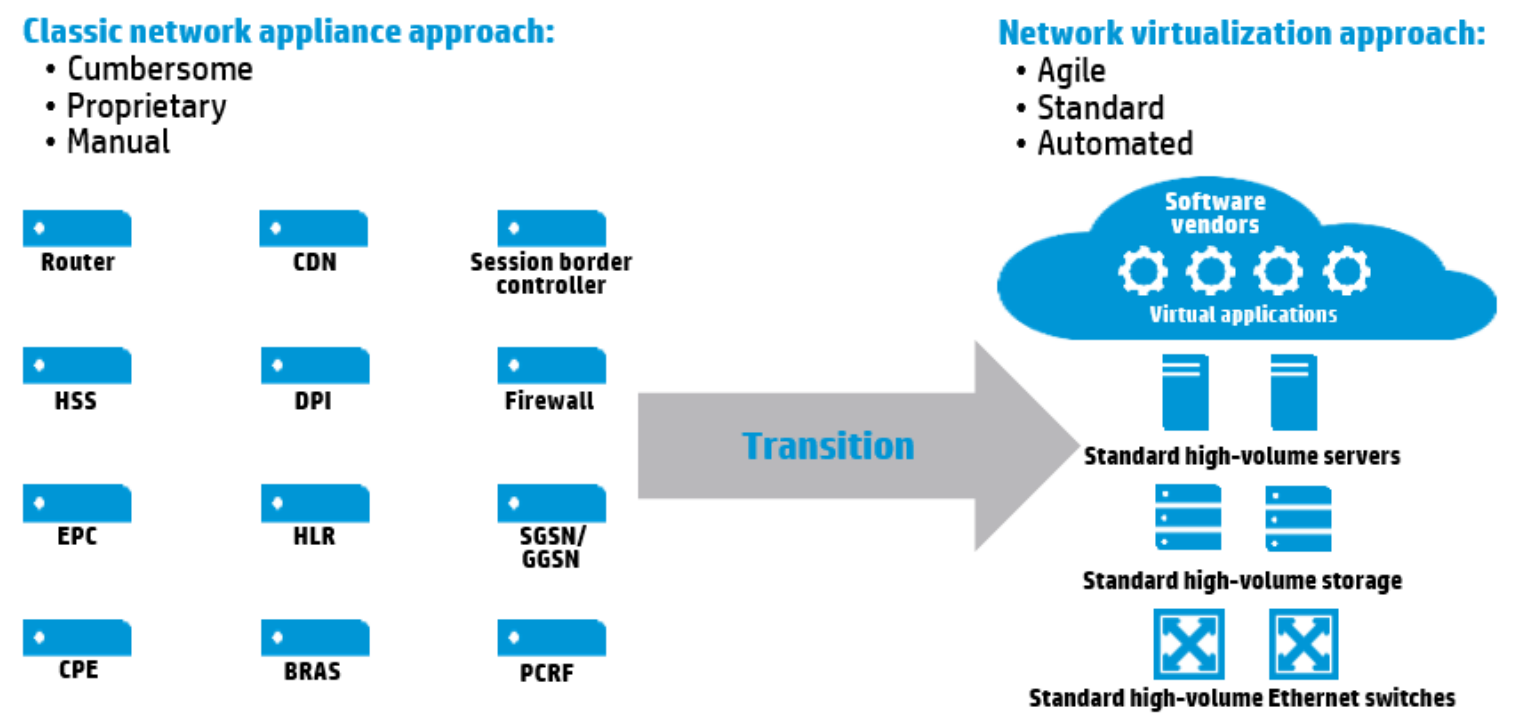

Figure 2 - From the Classical to a NFV approach (Source: Edlund, 2014)

\section{NFV Taxonomy}

As described in Figure 2, NFV replaces proprietary hardware network elements with software running on high-volume industry-standard servers, storage and network. NFV is a natural extension to the virtualization trend that has widespread adoption in the IT world, wherein virtual machines have replaced physical CPUs. Borrowing further from developments in cloud computing, virtualized network elements may be housed in IT data centres (rather than more expensive, specialised exchanges) in order to gain further economies of scale.

A Network Function (NF) is a building block within an telco's network infrastructure. NFs have well defined external interfaces and a well-defined purpose or behaviour. In practical terms, a NF is often a network node.

A Virtual Network Function (VNF) provides exactly the same functional behaviour and interfaces like the equivalent NF, but is deployed as software inside the NFV-Infrastructure.

The NFV-Infrastructure (NFV-Infra) is the totality of all hardware and software components which build up the environment in which VNFs are deployed, managed and executed. The 
NFV-Infrastructure can span across several locations or data centres. The network providing connectivity between these locations is part of the NFV-Infrastructure.

The $N F V$-Orchestrator $(\mathrm{NFV}-\mathrm{O})$ is software to operate, manage and automate the distributed NFV Infrastructure. The NFV-O has control and visibility of all VNFs running inside the NFV-Infra. The NFV-O provides a Graphical User Interface (GUI) and external NFV-Interfaces to the outside world to interact with the orchestration software. The NFVOrchestrator makes use of the NFV-Operating System to perform cloud operation and automation, thus extending the basic capabilities of the NFV-OS.

\section{NFV - Stages of Deployment}

Various stages of NFV deployment are possible and are shown in Figure 3.

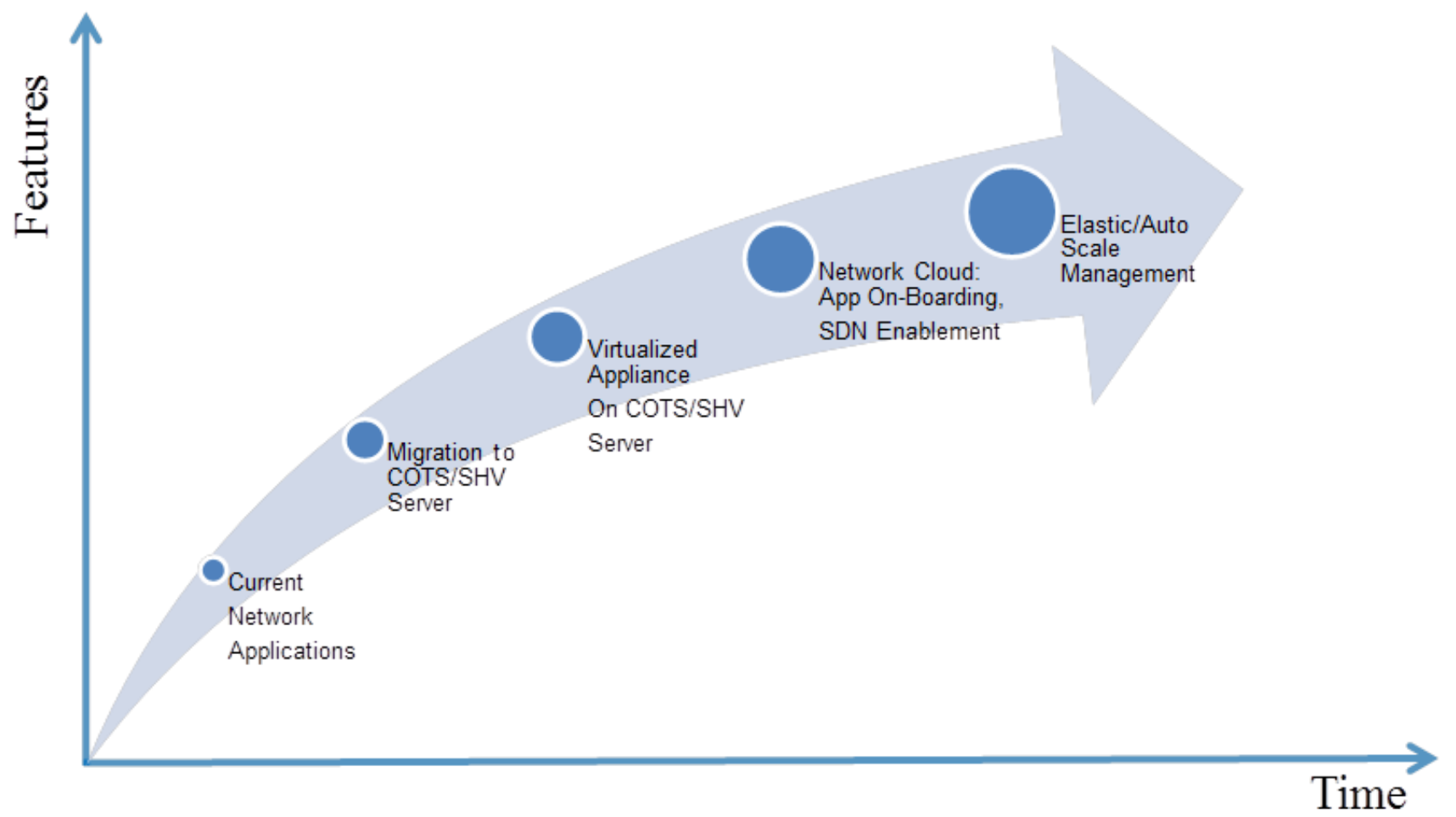

Figure 3: Various Stages of NFV Deployment (Adapted from Edlund, 2014)

Starting from the left, current network applications (or NFs) can be migrated to standard, high-volume (SHV) or commercial off-the-shelf (COTS) industry-standard servers, storage and switches. Next, NFs can be virtualised into software to become VNFs (sometimes referred to Apps) running on the SHV/COTS hardware. SDN Enablement occurs through the use of virtualisation controllers and NFV-Orchestrators, which can evolve to support the real-time performance requirements of telecom network functions. 


\section{Advantages of NFV}

NFV Advantages include the:

1. Faster deployment, relocation, upgrading, and turn-off of both networking and valueadded services - this is the ability to flexibly locate the NF where it is most effective or less expensive

2. More reasonable scaling of COTS (commercial off-the-shelf) equipment - which has a better price-performance ratio and more widespread availability in comparison to specialised, proprietary communications hardware.

3. Consolidation potential or the ability to combine multiple network functions (NFs) as VNFs on a single hardware platform.

\section{NFV Implementation Considerations}

There are a number of implementation considerations with NFV.

Portability/Interoperability. To maximise choice and economic benefit, an NFV implementation must have the ability to load and execute VNFs in geographically multiple, standardised datacentre environments, provided by different vendors. Portability also allows for the freedom to optimise the location and required resources of the virtual appliances without constraints. There could be some NFs that are strictly location dependent. However, virtualising such NFs will still lead to greater portability of VNFs within that location (e.g. data centre). It is important to probe $w h y$ the NF is location dependent and ensure a solid rationale exists.

Performance. NFV implementations must consider possible effects on latency, throughput and processing overhead and ensure they are minimized. (Choisi et al, 2012) believe that network control functions, as well as data/user plane functions can successfully be virtualised, and this has been evidenced in multiple HP proof of concepts as well.

Migration and Compatibility with legacy systems NFV implementations must coexist with telcos' legacy network equipment, element \& network management systems, and existing IT orchestration systems for NFV orchestration and IT orchestration to converge. The NFV architecture must support a migration path from today's proprietary physical network appliance based solutions to more open standards based virtual network appliance solutions.

Management and Orchestration. To reduce the cost and time to integrate new virtual appliances, standard management interfaces are required. Software Defined Networking (SDN) further extends this to the control of the forwarding behaviours of physical switches 
using SDN. This can be established at the proof of concept phase or through a study of the requisite northbound (VNF to NFV-O) interfaces.

Automation. NFV operations can only scale if all of the critical NFV-O functions can be automated. This should be included in the proof of concept test cases.

Security \& Resilience. NFV is an opportunity to improve the network resilience and availability by allowing network functions to be recreated on demand after a failure. The hypervisor and its configuration must be kept secure. Security certified hypervisors and virtual appliances are required.

Network Stability. A VNF and NF share the same network stability considerations. Care should be taken when virtual functions are relocated, or during re-configuration events, such as during failover scenarios.

Simplicity. VNFs will be simpler to operate than those that exist today due to the portability and consolidation opportunities. The focus should remain on the simplification of network architecture and operations to obtain ongoing cost savings.

Integration. In short, the ecosystem needs to come together at several levels - through the integration of multiple virtual appliances onto existing industry standard high volume servers and hypervisors, with the ability to "mix \& match" servers, hypervisors, and virtual appliances from different vendors without incurring significant integration costs and avoiding lock-in. The ecosystem must offer integration services and maintenance and thirdparty support as well as mechanisms to validate new solutions. Stakeholders in the ecosystem include OEM chip manufacturers (e.g. Intel and ARM); Network equipment Providers (NEPs - e.g. Ericsson, Nokia Networks, Juniper and Alcatel-Lucent); Independent Software Vendors (e.g. CheckPoint, Riverbed, F5), hardware server/storage/switch vendors (e.g. HP), system integrators (e.g. HP) and critically, the telcos themselves.

\section{Software Defined Networking}

SDN replaces standardized networking protocols with centralized software applications that configure all network elements. It requires the decoupling of network control and data plane functions, which is enabled by NFV or through the design of the NF. SDN promises to reduce the complexity of distributed networking control protocols with the simplicity of programming an omniscient controller.

SDN Advantages include the:

1. Easy experimentation with new ideas - ability to dynamically reconfigure the network on the fly to adapt it to instant demands for applications and services 
2. The more rapid development of software in comparison to protocol standardization and hardware development

3. Simplification of network maintenance due to centralized orchestration - ability to swiftly deploy, relocate and upgrade new features.

In a practical sense, a telco can instantiate a new network application / service using SDN principles to route or switch core network traffic flows between virtualised applications, enabling different services to be chained in the preferred order, achieving faster time to market. According to (GigaOm, 2014), the top 5 applications for open-source SDN are:

1. Network Optimisation

2. Security Services

3. Network Monitoring and Analytics

4. Network Automation

5. Policy Management

\section{The Relationship between SDN, NFV and an Open Approach}

Figure 4 visually outlines the relationship between SDN, NFV and open innovation.

NFV creates the operational flexibility, reduction in power and space requirements to address the supply-side issues identified above. Unit costs of NFs are reduced through the move to COTS hardware and IT (as opposed to network) level management of those functions. Moreover, utilisation is improved through virtualisation. SDN creates the necessary control abstractions to enable time to market and innovation cycle time reductions - which lowers management costs of end-to-end networks. Open innovation stimulates the supply market further through new permutations and combinations of cooperation and competition. For example, Linux and Apache have established themselves as viable alternatives to commercial software. It is reasonable to expect NFV and SDN equivalents, perhaps OpenStack (from the OpenStack Foundation) and OpenDaylight (from The Linux Foundation), to do so as well. 


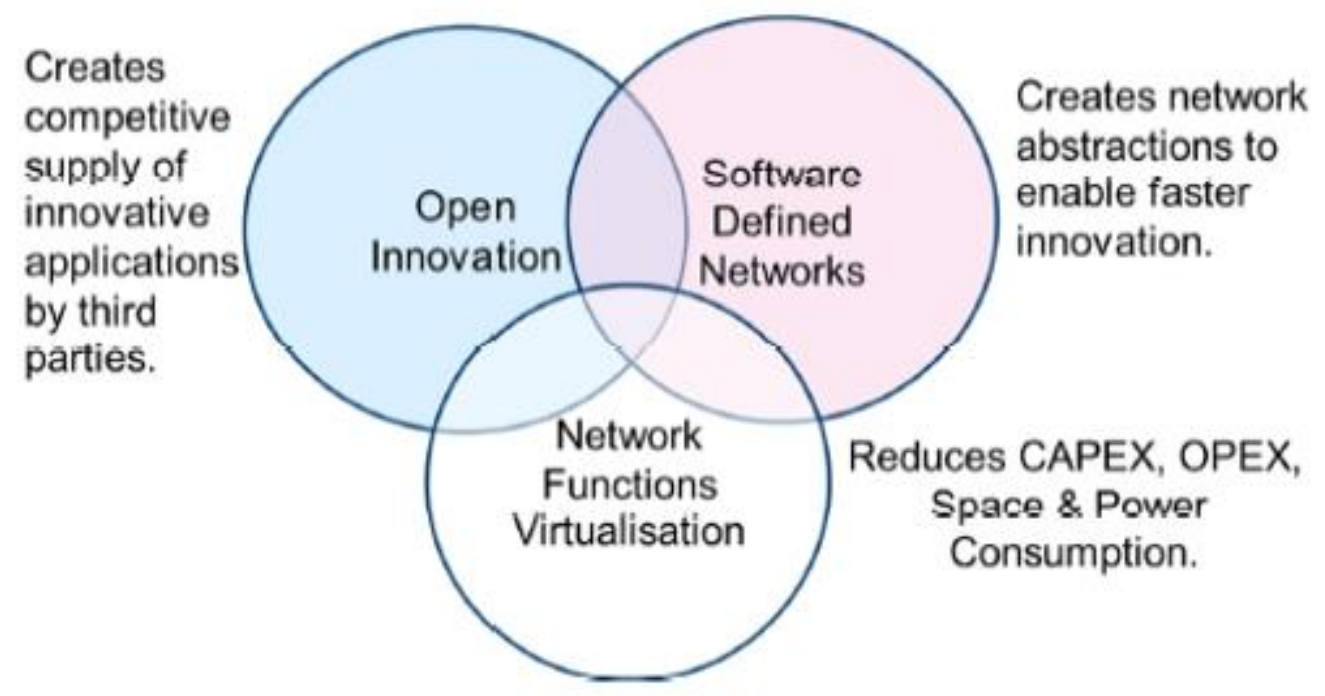

Figure 4: Relationship between SDN, NFV and Open Innovation (Source:

Edlund, 2014)

\section{NFV \& SDN Ecosystem}

The NFV ecosystem consists of the following players.

1. Telecom Network Operators or Telcos

2. Independent Software Vendors

3. Hardware (Server, Storage, Network) Vendors

4. Original Equipment Manufacturers - such as chipset vendors

\section{The Addressable Market}

Driven by the thriving ecosystem that has already formed, SNS Research estimates that the SDN and NFV will account for nearly \$4 Billion in investments globally in 2014. Despite the implementation considerations above with legacy networks, SNS Research estimates further growth at a CAGR of nearly $60 \%$ over the next 6 years. (SNS, 2013)

SDN and NFV investments are estimated to save wireless and wire-line service providers up to $\$ 32$ billion in annual capital expenditure by 2020. (SNS, 2013) Another research study said that $50 \%$ of operator respondents expect SDN to be implemented in mobile within five years. The (keener) 50\% expected it to be implemented in the next one to two years. (Informa Telecoms, 2014)

\section{Primary Research:}

Based on primary HP research involving the interview of 50 CIOs and CTOs of Tier $1 \& 2$ telcos, it is evident that NFV is a key trend. A third of those interviewed regarded NFV as the 
most important trend affecting their role above advances in mobility, cloud computing and software-defined networking technologies. 93\% expect NFV to be a major factor within 3 years. $(\underline{H P}, 2014)$

\section{Primary Research - Key business drivers}

The HP research above indicates that NFV is regarded as a technology that will help create new revenue and improve the telco's positioning vis-à-vis over-the-top (OTT) players. However, the demand for proof of concepts has focused on the supply-side economics. These include the reduced need for capital and operational expenditure, the opening up the network to innovation from a larger supplier group, and the shortening of innovation cycles, with the consequent lowering of risk when rolling out new services.

As NFV is based on open standards, telcos now have a choice of vendors for network functions - both the software and the use of lower cost industry-standard server, storage and networking hardware.

\section{Primary Research - HP Proof of Concepts}

HP's proof of concepts (PoCs) with leading CSPs include the virtualisation of several network functions. These include:

1. Customer Premises Equipment (CPE)

2. IPsec Termination

3. Broadband Remote-Access Servers (B-RAS)

4. Evolved Packet Core (EPC)

5. Content Delivery Networks (CDN)

6. Multimedia Services Environment

7. IP Multimedia Subsystem (IMS)

8. Next Generation IN (NGIN)

Through these, and other PoCs, HP has found that using off-the-shelf hardware can reduce the capital intensity of network appliances by up to $35 \%$, while leveraging open-source software can save up to another $35 \%$.

\section{Timeline of Adoption}

In 2013 and early 2014, early adopters have been experimenting and defining single function use cases with NFV. In 2014, it is expected that new and refreshed network software will start to be available in virtualised variants. Expect to see a number of PoCs in the labs in 
major service providers. (SDN Central, 2014) The volume rollouts of such technologies are expected in 2015 and beyond though more progressive telcos will adopt it sooner.

Conscious of potential virtualisation overhead, several telcos have begun their investigation by virtualising non-real time network functions such as store and forward messaging systems. Successful proof of concepts of network functions that are closer to real-time indicate that the technology is more broadly applicable.

In a BrightTALK webinar in April 2013, Dr Prodip Sen (Director, Network Architecture, Verizon Network \& Technology and Chair, ETSI NFV ISG) listed additional examples of where NFV can be applied: (Sen, 2013)

1. Application-level optimization: CDNs, cache servers, load balancers, application accelerators

2. Mobile networks: HLR/HSS, MME, SGSN, GGSN/PDN-GW, base station, EPC

3. Home environment: home router, set-top-box

4. Security functions: firewalls, intrusion detection/protection systems, virus scanners, spam protection

5. Tunneling gateway elements: IPSec/SSL VPN gateways

6. Traffic analysis/forensics: DPI, QoE measurement

7. Traffic monitoring, service assurance, SLA monitoring, test and diagnostics

8. NGN signaling: SBCs, IMS

9. Converged and network-wide functions: AAA servers, policy control and charging platforms

10. Switching elements: BNG, CG-NAT routers

Different service providers have different priorities and hence the sequence of virtualising NFs will differ.

\section{The role of standards}

Standards bodies, such as European Telecommunications Standards Institute (ETSI), Open Networking Foundation (ONF), Alliance for Telecommunications Industry Solutions (ATIS) TeleManagement Forum (TM Forum) and Internet Engineering Task Force (IETF) will play an essential, market-making, role in ensuring that NFV and SDN standards and specifications meet the needs of telcos. For example, NFV vendors will need to prove that a virtual network is just as secure as a physical network. (SDN Central, 2014) Standards bodies will also ensure that NFV and SDN retain an open architecture. 


\section{European Telecommunications Standards Institute}

The ETSI NFV initiative was launched in January 2013 to facilitate a technical, architectural and business community to accelerate the industry's move to NFV. This is a prestandardisation effort that brings together most global Tier-1 telcos, equipment vendors and IT vendors. Over 95 companies and 250 participants are involved. Significantly, 20 of the world's top telcos have created the ETSI standards that are the underpinnings for NFV.

As a result, ETSI has developed high-level specifications encompassing NFV terminology, requirements, end-to-end architecture and use cases. These use cases are detailed in the reference (ETSI 2013) below.

In addition to an ETSI NFV steering committee, there are working groups for infrastructure, software architecture, management and orchestration and proof of concepts, among other areas.

\section{Open Networking Foundation}

The ONF is a non-profit industry grouping, founded in 2011, that is advancing the role and standardisation of software-defined networking (SDN) architecture. For example, ONF is standardising the OpenFlow protocol, which ensures global coordination of network devices and communication between the control and data planes of supported network devices. ONF is also looking at traffic steering and service chaining, to ensure applications can communicate their requirements to the network and get notified of network conditions that impact application performance.

\section{Alliance for Telecommunications Industry Solutions (ATIS)}

ATIS is another industry consortium that is focused on SDN. ATIS drives its viewpoints and standards requests through other industry bodies, such as the $3^{\text {rd }}$ Generation Partnership Project for mobile. ATIS TOPS (Technology \& Operations Council) teams include those for telecom SDN use cases and Operations Support Systems (OSS).

\section{TM Forum}

TM Forum has traditionally had a strong operations support systems focus. In the NFV and SDN context, the TM Forum Catalyst projects are defining assurance and fulfilment frameworks.

\section{Internet Engineering Task Force}

The IETF's I2RS Working Group is also working on service chaining and SDN (IETF 2014). 


\section{Conclusions}

NFV and SDN have captured imagination and momentum - globally and in Australia. While NFV offers to immediately lower the capital and operational cost base by implementing a growing set of previously closed network platforms and functions on standard IT hardware, SDN will improve utilisation of network assets and allow lower-cost experiments to flex and scale capacity to improve network services and introduce new innovations. Some disruption to the traditional telco landscape can be expected to better address the dichotomy between a telco's cost structure and revenue trajectory.

\section{Acknowledgements}

I would like to thank the following colleagues for reviewing this document and for their useful suggestions:

1. Jeff Edlund, WW Communications and Media Solutions, Chief Technology Officer, HP

2. Julia Mason-Ochinero, WW Communications, Media \& Entertainment Marketing Leader, HP

3. Marie-Paule Odini, Communications, Media \& Entertainment, Office of the CTO, HP

\section{References}

ACMA. 2013. 'Australia's mobile digital economy - ACMA confirms usage, choice, mobility and intensity on the rise', Australian Communications \& Media Authority, http://www.acma.gov.au/theACMA/Library/Corporate-library/Corporatepublications/australia-mobile-digital-economy. Retrieved 7 May 2014.

Andreessen, Marc. 2011. 'Why Software Is Eating The World', Wall Street Journal, 20 Aug 2011, http://online.wsj.com/news/articles/SB1000142405311190348090457651225091562946 o. Retrieved 7 May 2014.

ATIS (Alliance for Telecommunications Industry Solutions). 2014. http://www.atis.org/about/index.asp. Retrieved 3 May 2014.

Bingemann, M. 2013. 'Vodafone splurges on network to win back customers and restore brand', The Australian, 19 Feb 2013, http://www.theaustralian.com.au/technology/vodafone-splurges-on-network-to-winback-customers-and-restore-brand/story-fn4iyzsr-1226580662375\#mm-premium, Retrieved 1 May 2014.

Bingemann, M. 2014. 'Optus gears up for battle', The Australian, 19 April 2014, http://www.theaustralian.com.au/business/optus-gears-up-for-battle/story-e6frg8zx1226889442241, Retrieved 1 May 2014. 
Chiosi, M. (AT\&T); Clarke, D., Willis, P., Reid, A., (BT); Feger, J., Bugenhagen, M., Khan, W., Fargano, M. (Century Link); Cui, C., Deng, H. (China Mobile); Benitez, J. (Colt), Michel, U., Damker, H. (Deutsche Telekom); Ogaki, K., Matsuzaki, T. (KDDI); Fukui, M. Shimano, K. (NTT); Delisle, D., Loudier, Q., Kolias, C. (Orange); Guardini, I., Demaria, E., Minerva, R., Manzalini, A. (Telecom Italia); López, D., Salguero, F.J.R. (Telefónica); Ruhl, F. (Telstra); Sen, P. (Verizon). 2012. 'Network Functions Virtualisation, An Introduction, Benefits, Enablers, Challenges \& Call for Action', SDN and OpenFlow World Congress, 22-24 October 2012, Darmstadt-Germany, http://portal.etsi.org/nfv/nfv white paper.pdf, Retrieved 1 May 12014.

GigaOm. 2014. 'SDN, NFV and Open Source - The Operator's View', Mar 2014, http://www.opendaylight.org/publications/sdn-nfv-and-open-source-operators-view, Retrieved 21 May, 2014

Edlund, J. 2014. Presentation on Network Functions Virtualisation, 'The New Business of the Network for CSPs', April 2014. bit.ly/1h7OA4h \#HPCMS.

ETSI. 2013. ETSI Industry Specifications Group (ISG) Network Functions Virtualisation, ETSI GS NFV 001 v1.1.1, October 2013, 'Network Function Virtualisation Use Cases', http://www.etsi.org/deliver/etsi gs/NFV/001 099/001/01.01.01 60/gs NFVo01v0101 o1p.pdf, retrieved 3 May 32014.

Hewlett-Packard. 2014. NFV Survey of 50 CIOs and CTOs from Tier 1 \& 2 operators, January 2014, Survey conducted by Coleman Parkes Research on behalf of HP in Europe, Middle East, Africa, Asia Pacific, North America and Central and Latin America.

IDC. 2014. 'Worldwide Telecommunications Predictions 2014', www.idc.com/getdoc.jsp?containerId=IDC P29872

IETF (Internet Engineering Task Force). 2014. https://datatracker.ietf.org/doc/draft-bitari2rs-service-chaining/ retrieved 22 June 2014

Informa Telecoms \& Media research. 2014. Quoted in www.ospmag.com/issue/article/AreYou-Hungry-For-SDN

Open Networking Foundation. 2014. https://www.opennetworking.org/, retrieved 3 May 3 2014.

Ovum. 2013. Consumer OTT VoIP Outlook 20013-18, November 2013.

Ramli, D. 2014. 'Telstra will keep investing in mobile', Australian Financial Review, http://www.afr.com/p/business/companies/telstra will keep investing in mobile Ra Kiz6AEH689yQpHYPqU3H, Retrieved 1 May 12014.

SDN Central. 2014. 'SDN and NFV Technology Trends to Watch in 2014', 6 January 2014. www.sdncentral.com/news/sdn-nfv-technology-trends-watch-2014/2014/01/

Sen, P. 2013. 'Defining NFV: Network Functions Virtualisation', https://www.brighttalk.com/webcast/6985/69053 Retrieved 2 May 22014.

SNS Research. 2013. 'The SDN, NFV \& Network Virtualization Bible: 2014 - 2020', October 2013, www.snstelecom.com/the-sdn-nfv-network-virtualization-bible

TeleManagement Forum. 2014. www.tmforum.org, retrieved 3 May 32014.

Cite this article as:

Sundar Iyer. 2014. Virtualisation of Network Functions and the SDN. Australian Journal of Telecommunications and the Digital Economy, Vol 2, No 2, Article 41.http://doi.org/10.7790/ajtde.v2n2.41. Published by

Telecommunications Association Inc. ABN 34732327 053. http://telsoc.org 